OAI-PMH: http://www.indteca.com/ojs/index.php/Revista Scientific/oai

Artículo Original / Original Article

\title{
La modelación, un recurso pedagógico para el pensamiento numérico y el aprendizaje significativo
}

\author{
Autora: Deisy Yasmine González Rojas \\ Secretaría de Educación Distrital, SED \\ deisyyasmineg@gmail.com \\ Bogotá, Colombia \\ https://orcid.org/0000-0002-8667-9194
}

\section{Resumen}

El proyecto pedagógico y matemático, esbozado en un estudio de investigación-acción, se cimentó en la intención de integrar conocimientos para la simulación de actividades cotidianas como la compra y venta de artículos básicos de la canasta familiar, coadyuvando en la apropiación, dinamización y enriquecimiento de contenidos propiamente dichos del pensamiento numérico, con estudiantes de Educación Básica Primaria, de un colegio de carácter público, de Bogotá; la intervención docente fue planeada, ejecutada y evaluada anualmente. El trabajo investigativo, tuvo una orientación mixta de tipo descriptiva, desarrollado con una población de estudiantes, de grados segundo, tercero, cuarto y quinto. La recolección y sistematización de información se realizó por medio de la técnica de observación directa y el procesamiento estadístico de los datos, derivados de un instrumento de evaluación, diseñado para que los educandos plasmaran sus propias apreciaciones y aprendizajes, luego de desarrollar y finalizar las actividades, con miras a innovar las prácticas en el aula. Las competencias matemáticas básicas, estimuladas y potenciadas, desde el pensamiento numérico, en el trabajo investigativo, se direccionaron por medio de acciones propias del contexto, simuladas en el espacio escolar, de manera creativa, logrando aflorar el reconocimiento de la relevancia del manejo y dominio numérico, en los colegiales. ambiente.

Palabras clave: investigación; aprendizaje; matemáticas; creatividad;

Cómo citar este artículo:

González, D. (2021). La modelación, un recurso pedagógico para el pensamiento numérico y el aprendizaje significativo. Revista Scientific, 6(19), 102-121, e-ISSN: 2542-2987. Recuperado de: https://doi.org/10.29394/Scientific.issn.2542-2987.2021.6.19.5.102-121

Fecha de Recepción: 16-09-2020
Fecha de Aceptación:

17-12-2020
Fecha de Publicación: 05-02-2021 
OAI-PMH: http://www.indteca.com/ojs/index.php/Revista Scientific/oai

Artículo Original / Original Article

\title{
Modeling, a pedagogical resource for number thinking and meaningful learning
}

\begin{abstract}
The pedagogical and mathematical project, outlined in an actionresearch study, was based on the intention of integrating knowledge for the simulation of daily activities such as the purchase and sale of basic items of the family basket, helping in the appropriation, dynamization and enrichment of contents proper of the numerical thought, with students of Primary Basic Education, of a public school, of Bogota; the teaching intervention was planned, executed and evaluated annually. The investigative work had a mixed descriptive orientation, developed with a population of students, from second, third, fourth and fifth grades. The collection and systematization of information was carried out through the direct observation technique and the statistical processing of the data, derived from an evaluation instrument, designed for the students to express their own appreciations and learning, after developing and completing the activities, with a view to innovating practices in the classroom. The basic, stimulated and enhanced mathematical competencies, from numerical thinking, in the investigative work, were directed through actions typical of the context, simulated in the school space, in a creative way, achieving the recognition of the relevance of management and mastery numerical, in schoolboys.
\end{abstract}

Keywords: investigation; learning; mathematics; creativity; environment.

\footnotetext{
How to cite this article:

González, D. (2021). Modeling, a pedagogical resource for number thinking and meaningful learning. Revista Scientific, 6(19), 102-121, e-ISSN: 2542-2987. Recovered from: https://doi.org/10.29394/Scientific.issn.2542-2987.2021.6.19.5.102-121
}

Date Received: 16-09-2020
Date Acceptance:

17-12-2020
Date Publication: 05-02-2021 


\section{Introducción}

El punto de partida del estudio investigativo, es la observación directa en el aula de clase, de la falta de motivación, durante el desarrollo de actividades matemáticas, propuestas y orientadas para la aprehensión conceptual básica, de contenidos asociados directamente a la aritmética; el poco interés por realizar ejecuciones operatorias, de tipo numérico, en los estudiantes del último grado de Educación Básica Primaria, promueve el accionar pedagógico, con miras a mitigar la apatía y a potenciar el aprendizaje significativo, de los contenidos concernientes al pensamiento numérico.

Como primer escenario escolar, se estima el aula de clase, convirtiéndola en un medio cambiante de aprendizaje, por tanto, se convierte en una tienda, en una miscelánea, donde hay intercambio de roles y modelación de situaciones fundamentales y diarias, que requieren del empleo de diversas habilidades, no sólo cognitivas, sino ciudadanas.

En tal sentido, Villa-Ochoa, Castrillón-Yepes y Sánchez-Cardona (2017): manifiestan que "cada manera de hacer modelación se materializa en una tarea o situación y en un ambiente de clase" (pág. 221); es así que, el diseño de una estrategia didáctica que pretende coadyuvar en la asimilación eficaz de conceptos numéricos fundamentales, en efecto canaliza actividades interrelacionadas y a la vez concatenadas que, dinamizan los ambientes de aprendizaje y por ende, los conceptos numéricos, generando aprendizajes significativos en Educación Básica Primaria.

Asimismo, el desarrollo de habilidades y destrezas matemáticas, además de estimar la apropiación de los conceptos, deben ser éstos, recreados, en ambientes de aprendizaje que modelen la/s realidad/es del contexto inmediato, lo que facilita el reconocimiento y la apropiación de la relevancia de lo que se aprende, cómo y para qué, y el argumento del por qué se aprende.

Teniendo en cuenta que, desde los inicios de vida escolar se deben 
consolidar las bases de los aprendizajes diversos y de esta manera, estructurar constructos conceptuales, necesarios y útiles para la integración y expansión de los conocimientos con la realidad; el pensamiento numérico, no es la excepción, por ende, toda estrategia y/o actividad encaminada al propósito de lograr un acercamiento de los contenidos temáticos, con la cotidianidad, debe ser el eje de la acción docente.

El pensamiento numérico, es una conjunción de conceptos y competencias, que incluye y hace parte de otros que, a su vez conforman la complejidad de las matemáticas, propiamente dichas; por lo que, las matemáticas, no pueden ser asumidas como un compendio exclusivo de técnicas y algoritmos, que facilitan el uso mecánico de simbologías, términos y cantidades, ya que éstos también requieren de una apropiación, para su representación, interpretación, comprensión y empleo apropiado, en contextos determinados. Según Castro, Rico y Castro (1995), afirman que:

Creemos que la etapa infantil es de enorme trascendencia para la educación matemática posterior del niño. En ella se van a formar los conceptos básicos o primarios y los primeros esquemas sobre los que, posteriormente, se construirá todo el aprendizaje. Si estos esquemas básicos están mal formados o son frágiles, pueden llegar a impedir o a dificultar (en el mejor de los casos) el aprendizaje posterior (pág. 2).

Entre tanto, enarbolar la ilusión por el proceso de aprendizaje matemático, en los educandos de los primeros años de formación escolar, requiere de una intervención pedagógica que, proyecte la conexión de la aplicación conceptual, en la realidad.

Los juicios valorativos que presentan los estudiantes, asociados éstos a su percepción de la poca o nula aplicación de lo que se aprende en la escuela, estimulados incluso desde los núcleos familiares, por preconcepciones o concepciones estructuradas de sus familiares y/o cuidadores, se convierten en la base, para que el docente pueda promover el 
interés y por qué no, hasta pretender un apasionamiento por las temáticas concernientes focalizadas, al pensamiento numérico, que es uno de los pensamientos que se esboza desde los grados académicos iniciales.

El avance que se da durante los estudios complejiza los conocimientos pretendidos en la formación académica secundaria. Con respecto a lo antes mencionado, Forero (2020): afirma que "[...] las experiencias asociadas con la modelación matemática que permitan transitar por diferentes procesos [...] son una fuente de vivencias que promueven el desarrollo de procesos de matematización [...]" (pág. 77); de ahí que, el constructo matemático, para ser comprendido, se apoya de la transversalidad de sus estructuras temáticas, específicas.

En consecuencia, potenciar la comprensión conceptual matemática, exige el apoyo de prácticas matematizadas, éstas directamente correlacionadas con situaciones del contexto inmediato. La formación académica primaria, mayormente debe estar enmarcada en la realidad, ya que esta etapa de los colegiales promueve la aprehensión, motivada y enriquecida por los sentidos; además, la intervención del juego, la simulación y la modelación, facilitan las adquisiciones netamente conceptuales; de ahí que, transversalizar los contenidos y conceptos, puede consolidar aprendizajes.

Modelar el contexto, en el espacio escolar, cautiva el interés y por ende la atención de los menores y si a esta acción pedagógica, se le adiciona el uso creativo de elementos, la interacción en los procesos y la retroalimentación permanente, la enseñanza y aprendizaje se da en ambientes más dinámicos, perpendiculares e incluyentes.

Si el educando siente el agrado por aprender, éste vincula también en su proceso a su familia y/o cuidadores y no es el docente quien tiene que ligar el apoyo de casa, éste también se puede dar a través del entusiasmo, de quienes disfrutan el proceso de aprender. Conocedores de que el aspecto académico, es uno de los más enfocados por las políticas educativas actuales, 
éste demanda toda la atención posible, acudiendo a la diversidad que nos brinda la didáctica de las matemáticas.

Asimismo, Díaz (2018): expresa que "en América Latina, la educación para el desarrollo sustentable se centra en el aspecto académico [...]" (pág. 42); de ahí que, es uno de los enfoques docentes, se debe direccionar el propósito de coadyuvar en este progreso. El accionar docente en las primeras etapas de la formación académica en los escolares, puede llegar a esbozar una plataforma tan sólida, como flexible, para los aprendizajes que posibiliten una estructuración y concatenación de conceptos, mayormente complejos.

Los proyectos de aula extrapolados al entorno y ambiente escolar permiten el reconocimiento y mejoramiento de falencias en el proceso de enseñanza/aprendizaje, además que potencian habilidades, no solo disciplinares de las matemáticas propiamente dichas, sino otras, útiles para la vida. En ese mismo orden de ideas, D’Amore, Díaz y Fandiño (2011): sostienen que "si con la acción didáctica se pretende el desarrollo de competencias matemáticas por parte del estudiante, el currículo debe ser diseñado bajo este objetivo específico" (pág. 52); por tanto, la transversalidad resulta un apoyo.

Las actividades realizadas de manera grupal impulsan prácticas sociales vinculantes de aprendizajes que, concatenan con otros, de manera engranada y armónica, canalizando así, aprendizajes significativos. Desde los primeros grados de formación académica, los escolares deben orientar sus múltiples conocimientos al propósito de ser cada vez más independientes, no solamente en sus acciones, sino también en sus interpretaciones, por tanto, la actuación del docente resulta ser uno de los más y mayormente influyentes en tal emancipación.

Así pues, Acosta y Acosta (2019): manifiestan que "[...] para que el estudiante sea capaz de mostrar autonomía no solo se necesita que se fomenten estrategias tradicionales de cognición y metacognición sino 
profundizar con interés e ímpetu en las estrategias metodológicas [...]" (pág. 145). La innovación y la dinamización de los procesos matemáticos, en el contexto escolar, se promueven desde las ópticas cambiantes del docente. Por consiguiente, Cadenas (2017): sostiene que "el quehacer educativo no debe permanecer aferrado a ciertos paradigmas, debe aceptar nuevas perspectivas [...]" (pág. 290); por tanto, se entiende la enseñanza y el aprendizaje, como cambiantes y flexibles.

Por medio de la observación, del ejercicio efectuado en el aula de clase, en conjunción con los resultados obtenidos del instrumento de evaluación, diseñado para que los educandos del último grado académico de Educación Básica Primaria plasmaran sus apreciaciones, en relación con la implementación del proyecto de aula, y de la inquietud que generó en estudiantes de otros grados, resultó interesante proyectar la intencionalidad pedagógica a los grados: cuarto, tercero y segundo de Educación Básica Primaria.

A través de un proyecto de área, a fin de paliar las falencias presentadas en el área de matemáticas, desde edades más tempranas, en etapas escolares, a fin de incentivar el gusto por los conocimientos matemáticos, desde la transversalidad. De manera tal que, el accionar pedagógico, resulta pertinente y conveniente, cuando se trata de mejorar aspectos, que se aprecian como dificultades, por medio de la modelación.

El proyecto que inicia como de aula, para unas clases específicas de matemáticas, con la simulación de la dinámica cotidiana de una tienda, donde la mitad del curso juega el rol de vendedor y la otra de comprador, genera tanto impacto positivo, en el estudiantado, que luego, al año siguiente se convierte en un proyecto de área y multigrado, en donde cada escolar es un actor, precursor de su propio aprendizaje, de manera creativa.

Es así que, en el año lectivo 2013, se proyecta en el colegio distrital Miguel Antonio Caro, Institución Educativa de carácter público, el proyecto de 
aula: la tienda y las matemáticas, que además de ser un enlace para fomentar cocimientos matemáticos específicos, resultó un vínculo de enriquecimiento de habilidades y destrezas, diversas, ya que la modelación de la práctica cotidiana de la tienda, demandaba de la creatividad para diseñar y crear el ambiente de aprendizaje -parecido a una tienda- en el aula; dicho proyecto, para el año lectivo 2014.

Viéndose su alcance, se convirtió hasta el año lectivo 2019 en un proyecto de área, llamado: la tienda como recurso de aprendizaje en el MAC sigla de la institución- impactando cuatro de los cinco grados de Educación Básica Primaria, donde el grado quinto fue el encargado del diseño y organización del ambiente de aprendizaje, llevando incluso formatos de inventario de los artículos simulados para la venta, mientras que los grados cuarto y tercero.

Se prepararon las clases, para realizar cálculos de situaciones reales, que les sirviesen para realizar compras simuladas; los grados tercero y segundo, elaboraron el dinero didáctico -billetes y monedas- para la simulación de los procesos de compra y venta de artículos. El proceso desde el diseño hasta la implementación, realizado dos veces al año, arrojó como resultado final, un interés entre los educandos, por las actividades programadas e implementadas, las cuales procuraban dinamizar los saberes y el desarrollo al interior de las clases de matemáticas.

Las actividades llevadas a cabo en el proyecto de área, se asociaron a un proceso de evaluación continua, que como directriz tuvo el propósito de favorecer el mejoramiento del accionar docente, para así lograr optimizar la participación de los educandos; además, el instrumento empleado para el proceso evaluativo, que fue diligenciado y valorado por los estudiantes, razón por la que se vinculó desde grado segundo que ya su proceso de lectura y escritura es un poco más fluido, que el grado primero.

Los aspectos para tener en cuenta son: la organización de las 
actividades, la participación de ellos mismos, la evidencia de lo trabajado en clase, la actitud de ellos en el desarrollo de las actividades, la explicación de qué fue lo que más les gustó y qué aprendizajes consideraron obtener o emplear en las actividades ejecutadas, permitiendo una mirada holística del proyecto, aunado a la observación directa y permanente del docente organizador.

Para el Ministerio de Educación Nacional (1999): "cualquier proceso de construcción de conocimiento está mediado por un instrumento, ya sea material o simbólico [...] El conocimiento y el aprendizaje son, por su naturaleza situados, es decir, dependen fuertemente, en su construcción, de la especificidad del contexto" (pág. 28). De manera tal que, el empleo de las concepciones básicas del pensamiento numérico, fueron recreadas y asumidas, incentivando el reconocimiento de la relevancia del manejo del cálculo mental, operatoria básica y en la práctica simulada de la cotidianidad.

Las competencias matemáticas básicas, se direccionaron en el desarrollo del proyecto de área de matemáticas, en Educación Básica Primaria, desde el pensamiento numérico, por medio de, la estimulación de los saberes, para potenciarlos, a través de acciones propias del contexto, simuladas en el espacio escolar.

\section{Metodología (Materiales y métodos)}

Como punto de partida, en el año lectivo 2013 se identificaron en las clases de matemáticas, dificultades en las competencias propias del pensamiento numérico, en educandos de grado quinto de Educación Básica Primaria de colegio de carácter público distrital, lo anterior evidenciado en la falta de apropiación conceptual de los algoritmos aditivos elementales, su aplicación y su cálculo fluido en la resolución de situaciones cotidianas expuestas, por tanto se infirió en la falta de sentido y significado de las concepciones propiamente dichas de las operaciones matemáticas básicas. 
Es así como, en este mismo año académico, se decide diseñar un proyecto pedagógico de aula, para promover la apropiación y significación de la operatoria numérica, de manera lúdica y creativa, con estudiantes del grado quinto de Educación Básica Primaria, del colegio Miguel Antonio Caro IED, ubicado en la ciudad de Bogotá, Colombia.

La dinamización de los aprendizajes matemáticos, netamente del pensamiento numérico, se buscó potenciarlo desde el aula de clase, a través de la simulación de actividades concernientes a la rutina de los escolares, referentes a la compra y venta de artículos varios, especialmente de los componentes de la canasta familiar, además de aprovechar la ubicación y contexto circundante de la Institución Educativa, pues es una zona de comercio diversificado en muchos aspectos, que genera un ambiente de aprendizaje matemático, de fácil modelación en el espacio escolar.

De manera tal que, la modelación del contexto inmediato de los colegiales, en conjunción con sus rutinas diarias, observadas y vivenciadas, tanto en el trayecto hacia el colegio, como en sus prácticas socioeconómicas habituales, coadyuvaron en el propósito de generar ambientes de aprendizaje dinámicos, creativos y simulados en el aula de clase, para favorecer la potenciación de aprendizajes básicos de matemáticas, particularmente del pensamiento numérico.

Para aterrizar la perspectiva matemática, en el aula de clase, se solicitó por grupos de trabajo, que cada uno de éstos, se fijara e indagara cómo era el proceso que se daba en los negocios y/o tiendas que se encontraban alrededor del colegio y/o de sus casas, luego por medio de conversatorios, en las clases de matemáticas, se promovió un ejercicio de reflexión, asociado a la relevancia y pertinencia de las conceptualizaciones matemáticas elementales, propias del pensamiento numérico, situación que conllevó a la reflexión docente, de cómo podría darse un aprendizaje menos estricto.

Teniendo en cuenta, las peculiaridades propias de los educandos y su 
entorno, luego de recolectar información, por medio de los conversatorios, las observaciones directas en clase, los procesos reflexivos e interacciones direccionadas a comprender los tópicos generativos que darían la ruta metodológica y procesos, para los logros estimados, correlacionados con la implementación de conceptos numéricos básicos y la mejor ejecución operatoria, se estimó apropiar elementos de la Investigación-Acción (I.A.).

Los datos obtenidos permitieron ser valorados, de manera cualitativa y cuantitativa, de manera tal que, el estudio investigativo se proyectó con un enfoque mixto, a una muestra no aleatoria. El control de las variables se realizó, con el apoyo de la observación directa, en conjunción con el instrumento de evaluación, diseñado para la actividad, que se estructuró, enfatizando en el aspecto actitudinal de los escolares y su percepción de aprendizaje matemático.

Con los insumos obtenidos, por medio de las observaciones directas, los aportes de los escolares en los conversatorios y la interacción permanente en clase, se estructuró el plan de acción pedagógica, enriquecido continuamente con las contribuciones de los mismos educandos; por lo que, se planificó teniendo en cuenta la contribución de los menores del grado quinto, que con su entusiasmo lograron que el proyecto que se inició con y para el grado quinto, se extendiera a los cuatro grados de Educación Primaria, anteriores a éste.

Se estimó por clase, que se trabajara una situación matemática correlacionada con la compra y/o venta de algún/os artículo/s de consumo diario, al iniciar o finalizar el momento pedagógico y adicional, se realizara un trabajo organizativo, con miras al logro de la adecuación del aula de clase, como medio de modelación y ambiente diferente, para dinamizar diversos aprendizajes, enfatizando en el matemático.

Los educandos, se dividieron libremente en grupos de trabajo, de mínimo tres y máximo cuatro integrantes, para distribuirse las tareas extra- 
clase, como: la elaboración de carteles promocionales para los negocios o tiendas simuladas, la consecución de empaques, el recorte de figuras (para modelar la venta de artículos diversos, como técnicos, tecnológicos, ropa y zapatos), dinero pedagógico (billetes y monedas calcadas).

Las actividades, permitieron el desarrollo de múltiples habilidades, entre ellas las sociales, hasta lograr que los miembros de las familias de los escolares contribuyeran con el proceso de aprehensión matemática, al punto que, de manera escrita y personal se le manifestara a la docente, promover tales actividades a los otros grados, ya que los más pequeños en casa, también mostraban particular interés, por participar.

Después de la adecuación de los espacios, donde unos escolares participaron como compradores y los otros como vendedores, se efectuaron las acciones simuladas de compra y venta, respectiva, pegando en los cuadernos o portafolios de matemáticas, las compras y de quienes participaron de vendedores, el formato de inventario y ventas realizadas, para luego en las siguientes clases, fuesen empleados tales datos, para ejemplificar situaciones aditivas.

Tal actividad realizada al final del primer semestre académico fue retomada, para el segundo, pues manifestaron los estudiantes que querían realizar cambio de roles, debido al entusiasmo expresado y evidenciado, se tomó a consideración y se efectuó, según lo solicitado. Debido a lo suscitado, para el año lectivo 2014 se extendió el proyecto de aula a los grados cuarto, tercero y segundo de Educación Básica, ya como proyecto del área de matemáticas, donde con la colaboración de colegas docentes, se pudo estructurar de manera transversal, debido al grado de escolaridad.

Se enfatizó en el desarrollo de ciertas habilidades motrices, lógicomatemáticas y sociales. En el grado segundo, se trabajó el ejercicio de cálculo aditivo escrito y mental, con cantidades monetarias reales, en situaciones reales y cotidianas para muchos de los estudiantes, como la compra del pan 
para el desayuno con un billete de determinada denominación, su cambio respectivo, sus vueltas y sus faltantes.

En este grado, se realizaron actividades en las clases de artes, concernientes a la elaboración de dinero pedagógico, con cartulinas o papeles de colores, lo que involucró sus habilidades y destrezas motrices y de coordinación. En el grado tercero, también se estimó ejemplificar situaciones para resolver en los cuadernos y realizar cálculos mentales, de tipo aditivo, incluyendo la abreviación operatoria, por medio del empleo de la multiplicación, con situaciones también reales y del contexto inmediato.

Este grado estuvo a cargo de colaborar con el grado segundo, de elaborar dinero pedagógico, además de conseguir paquetes o envolturas de artículos, recortar otros, que servirían para la simulación del ambiente. Mientras que, el grado cuarto, colaboró con la elaboración de carteles promocionales de las tiendas varias, de venta de diferentes artículos, también ayudando al recorte de artículos; en este grado, se trabajó ejercicios de cálculo básico, enfatizando en el empleo de la multiplicación y la división, como operaciones que nos ahorran tiempo para realizar cuentas.

Por otra parte, los cursos del grado quinto, se distribuyeron tareas referentes a los inventarios de artículos e indagación de precios reales de artículos diversos, según las tiendas a modelar, pues este grado se dedicó a realizar la modelación de la venta de artículos a los demás grados, actividad distribuida durante el año lectivo, debido a la cantidad de cursos por grado, dos por grado con promedio de treinta y cinco estudiantes, cada curso.

El grado quinto, enfatizó su aprendizaje en el manejo de inventarios sencillos y en la ejecución de cálculos mentales, empleando varias operaciones matemáticas, a fin de optimizar la atención a los compradores simulados, incluyendo el empleo con criterio matemático de calculadoras elementales.

Observándose el entusiasmo de los escolares, con base en sus 
percepciones y apreciaciones plasmadas en el instrumento de evaluación, que se compartió en la siguiente clase, luego de la actividad principal (la simulación de la actividad en la tienda), cada educando consignó y valoró las actividades específicas y la general; tal insumo contribuyó a la toma de decisión de seguirse implementando, teniendo en cuenta los resultados obtenidos, en los siguientes años académicos, hasta el año lectivo 2019.

El grado preescolar y primero, dadas sus particularidades en el proceso de lectoescritura, en conjunción con el desarrollo de sus habilidades, los docentes de estos cursos estimaron encaminar actividades similares, dentro del desarrollo propio de sus actividades pedagógicas. Con la simulación de los procesos y ambientes de una tienda, en el aula de clase, se promovieron habilidades y destrezas en os educandos, para éstos resultó tan motivante como innovador, ya que la práctica simulada de la compra y venta de artículos requería de todo un esquema de organización, que involucraba su entusiasmo, creatividad y trabajo cooperativo, entre pares estudiantes, movilizando también, las ayudas de sus familiares y/o cuidadores.

Los resultados, plasmados en el instrumento de evaluación, generaron prácticas relevantes, incluyendo a la autoevaluación en el actuar para llevar a cabo las acciones atenientes a la compra y venta de artículos, con valores reales, que exaltó la participación y movilización de aprendizajes interdisciplinares y transversales, motivando a los otros/as compañeros/as de diferentes grados y cursos, al punto de lograr la extrapolación de las acciones pedagógicas, a otros grados de Educación Básica Primaria e incluso de sostenerse el proyecto durante seis años.

La transformación del proyecto de aula, a un proyecto del área de matemáticas, sembró en la mayoría de los escolares de primaria, la semilla del reconocimiento y valor de lo que se aprende en el área de matemáticas, asociado al pensamiento numérico, ya que vivieron el empleo y necesidad de éste, para las actividades cotidianas de compras y ventas, que son prácticas 
comunes e inherentes a la comunidad actual.

Debido a la amplitud del proyecto y su alcance pretendido, se tuvo en cuenta, el grado de escolaridad, para realizar las tareas; por ello, desde el grado segundo, hasta quinto, se enfocó el empleo de algoritmos básicos, para resolver situaciones matemáticas reales, con valores reales y con dinero (billetes y monedas) didáctico, adquirido en misceláneas y/o elaborado en clase, con cartulinas.

Independientemente de la temática a desarrollar en clase de matemáticas, se iniciaba o finalizaba, con una situación planteada para resolverla con material concreto y de manera simbólica en los respectivos cuadernos de apuntes o portafolios; adicionalmente a los refuerzos de operatoria básica, en las clases y su aplicación a eventos reales modelados, se promovió el enriquecimiento y potencialización de diversos talentos, que fue parte del propósito: la tienda, como recurso de aprendizaje significativo en el MAC -sigla del colegio-, nombre del proyecto.

La concatenación de acciones pedagógicas, su evaluación y reflexión, continua y constante, permitió que el entusiasmo y agrado por el desarrollo de las actividades se mantuviese y se incrementara, al punto de continuarse y proponerse para grados de Educación Básica Secundaria, lo cual, por motivos de pandemia, se dilató, sin embargo, es estimable su proyección e implementación en dichos niveles superiores, de formación, con miras a mejorar los aprendizajes y el agrado por lo que se aprende, en los diferentes pensamientos matemáticos y su consolidación en el entorno inmediato.

\section{Resultados (análisis e interpretación de los resultados)}

Con el instrumento de evaluación, del proyecto de área, denominado: la tienda como como recurso de aprendizaje significativo en el colegio, arrojó datos cuantitativos y cualitativos, de las variables independientes, cualitativas continuas, del pensamiento matemático: numérico y su empleo en situaciones 
modeladas y reales, que se estructuró de manera constante, con miras a mejorar el trabajo de investigación-acción, que se cimentó en la meta inicial propuesta y esbozada a lograr un reconocimiento y aprendizaje con sentido, significado y apropiación de conceptos propios del pensamiento numérico.

En los siete años lectivos, en los cuales se implementó el proyecto, se impactó a una población estudiantil mixta de 1759 colegiales, en rango de edades entre 7 años y 14 años, de condiciones socioeconómicas medio-bajas. Con el apoyo de los estadísticos de correlación de Pearson, para probar hipótesis bosquejadas, en correspondencia con el impacto positivo de la modelación, como uno de los recursos pedagógicos, que potencian el pensamiento numérico y el aprendizaje significativo, se apreció que es posible a partir del desarrollo de actividades simuladas, captar mejor la atención.

Gráfico 1. Evaluación referente al proyecto del área de matemáticas.

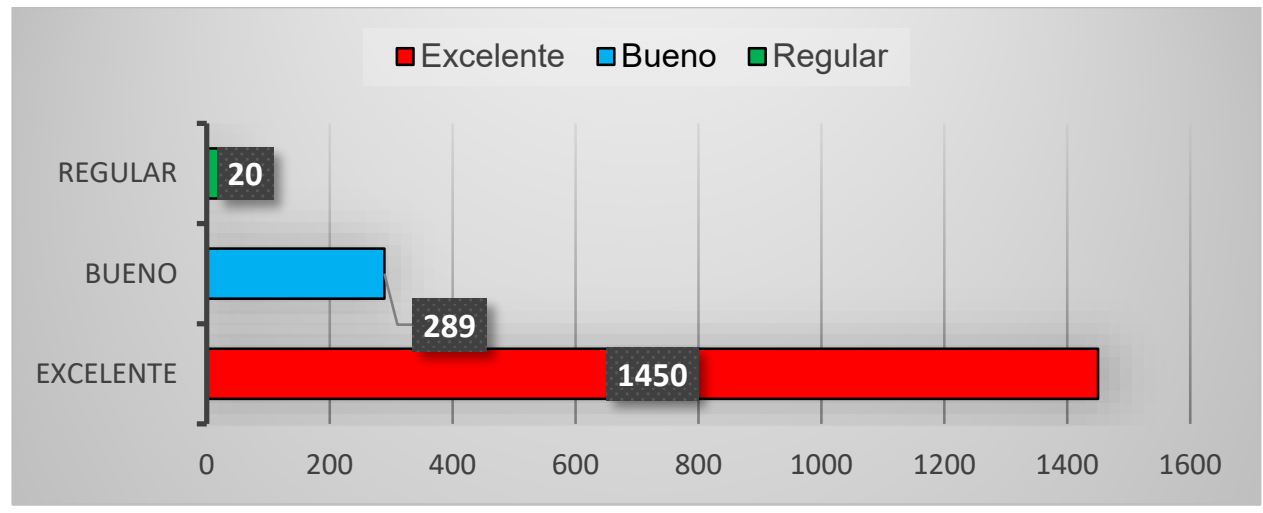

Fuente: La Autora (2020).

Al preguntar a los escolares por su percepción (1450) valoraron ésta como excelente, (289) buena y (20) regular, asociando los diversos momentos del desarrollo del proyecto de área de matemáticas, enmarcado en la metodología investigación-acción, realizado desde las clases del área, por siete años lectivos, desde el año 2013, hasta el año 2019, los resultados obtenidos, por medio del instrumento de evaluación escrito, permitió apreciar 
que, la mayoría de los estudiantes, valoraron en excelente el adelanto del proyecto, como se ve en el gráfico 1.

Es de resaltar que, la apreciación y valoración correspondiente de los educandos, referente al proyecto de área de matemáticas, sostenido por siete años lectivos, se logra luego de realizar un compendio los siguientes aspectos, tenidos en cuenta para la calificación y cualificación de éste: la organización de las actividades, la participación de los escolares y docentes, el trabajo en clase, los aprendizajes obtenidos y la actitud propia en y durante el proceso del proyecto.

De ahí que, el accionar de los participantes, con relación a la organización, fue considerado por la mayoría como excelente, al igual que la participación, el trabajo en clase, los aprendizajes logrados y el entusiasmo por el desarrollo de las actividades y las tareas que las comprendieron, dándose un ambiente educativo modelado y creativo, para la consolidación y potenciación de saberes.

\section{Conclusiones}

La imagen de las matemáticas resulta en ocasiones como negativa e inservible, sin sentido, para quienes las aprenden, pues su inmersión en la cotidianidad y su pertinencia no es vislumbrada de manera inmediata, por ende, como docentes, más de esta área fundamental, es un desafío que se vea y perciba la conveniencia de sus conceptualizaciones, desde los primeros grados de formación académica, desmitificando su dificultad, inutilidad y poca comprensión conceptual.

Por tanto, la intervención pedagógica, oportuna desde la mirada de la investigación cualitativa, Investigación-Acción, apoya además de la reflexión crítica, la focalización de situaciones problemáticas que posibilitan actos cambiantes o con propósitos a la mejora e innovación didáctica; es así que, la modelación puede facilitar el uso de saberes propios del pensamiento 
numérico, para lograr un aprendizaje significativo, dinámico, con sentido, que coadyube en la apropiación de otros compendios conceptuales, mayormente estructurados y complejos, no menos, ni mayormente importantes, ya que cada aprendizaje trae su propia dinámica y consigo resulta una integral transversalidad.

\section{Referencias}

Acosta, M., \& Acosta, M. (2019). Formación del Pensamiento Reflexivo para el Logro de un Aprendizaje Significativo de la Física en Estudiantes Universitarios. Revista Scientific, 4(12), 141-161, e-ISSN: 2542-2987. Recuperado de:

https://doi.org/10.29394/Scientific.issn.2542-2987.2019.4.12.7.141-161 Cadenas, Y. (2017). Estrategias Instruccionales Alternativa para la Comprensión Lectora. Revista Scientific, 2(4), 283-301, e-ISSN: 25422987. Recuperado de: https://doi.org/10.29394/scientific.issn.2542$\underline{2987.2017 .2 .4 .16 .283-301}$

Castro, E., Rico, L., \& Castro, E. (1995). Estructuras Aritméticas Elementales y su Modelización. México, D.F.: Grupo Editorial Iberoamérica, S.A. de C.V.

D’Amore, B., Díaz, J., \& Fandiño, M. (2011). Competencias y Matemática. ISBN: 978-958-20-0939-7. Bogotá, Colombia: Cooperativa Editorial Magisterio.

Díaz, B. (2018). Patios Productivos como Estrategia para la Consolidación de la Educación Ambiental. Revista Scientific, 3(10), 40-60, e-ISSN: 2542-2987. Recuperado de:

https://doi.org/10.29394/Scientific.issn.2542-2987.2018.3.10.2.40-60 Forero, A. (2020). Procesos de modelación matemática en formación de profesores de matemáticas. Revista de la Facultad de Ciencias Universidad Nacional de Colombia, 9(2), 66-79, e-ISSN: 2357-5749. 
Recuperado de:

https://revistas.unal.edu.co/index.php/rfc/article/view/86884/75628 Ministerio de Educación Nacional (1999). Nuevas Tecnologías y Currículo de Matemáticas Lineamientos Curriculares. Santa Fe de Bogotá, Colombia: Cooperativa Editorial Magisterio.

Villa-Ochoa, J., Castrillón-Yepes, A. \& Sánchez-Cardona, J. (2017). Tipos de Tareas de Modelación para la Clase de Matemática. Espaço Plural, 36(1), 219-251, e-ISSN: 1981-478X. Recuperado de:

http://funes.uniandes.edu.co/11774/ 


\section{Deisy Yasmine González Rojas}

e-mail: deisyyasmineg@gmail.com

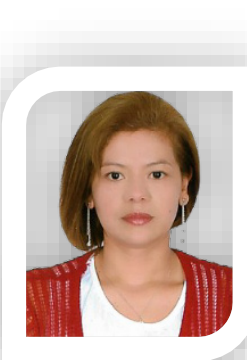

Nacida en Colombia, Departamento de Cundinamarca, municipio de Nocaima, el 15 de abril del año 1981. Doctorante en Gerencia Pública y Política Social; Doctora en Educación; Magister en Neuropsicología y Educación; Magister en Docencia Universitaria; Especialista en Docencia Universitaria; Licenciada en Educación y Promoción de la Comunidad con énfasis en Matemáticas; Bachiller Pedagógica; Me he desempeñado por más de veinte años, en el campo de la educación, como docente en educación preescolar, primaria, secundaria y media vocacional, también como directiva docente en el sector rural y urbano.

El contenido de este manuscrito se difunde bajo una Licencia de Creative Commons ReconocimientoNoComercial-Compartirlgual 4.0 Internacional 